\title{
Nuclear physics for the Re/Os clock
}

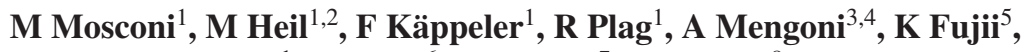 \\ C Domingo-Pardo ${ }^{1}$, G Aerts ${ }^{6}$, R Terlizzi ${ }^{7}$, R Gallino $^{8}$, U Abbondanno ${ }^{5}$, \\ H Álvarez ${ }^{9}$, F Alvarez-Velarde ${ }^{10}, \mathbf{S}$ Andriamonje ${ }^{6}$, J Andrzejewski ${ }^{11}$, \\ P Assimakopoulos ${ }^{12}$, L Audouin ${ }^{6}$, G Badurek ${ }^{13}$, P Baumann ${ }^{14}$, \\ F Bečvár ${ }^{15}$, E Berthoumieux ${ }^{6}$, F Calviño ${ }^{16}$, M Calviani ${ }^{17}$, D Cano-Ott ${ }^{10}$, \\ R Capote $^{3,18}$, C Carrapiço ${ }^{19}$, P Cennini ${ }^{4}$, V Chepel $^{20}$, N Colonna ${ }^{7}$, \\ G Cortes $^{21}$, A Couture ${ }^{22}$, J Cox ${ }^{22}$, M Dahlfors ${ }^{4}$, S David ${ }^{14}$, I Dillmann ${ }^{1}$, \\ W Dridi ${ }^{6}$, I Duran ${ }^{9}$, C Eleftheriadis ${ }^{23}$, M Embid-Segura ${ }^{10}$, L Ferrant ${ }^{24}$, \\ A Ferrari $^{4}$, R Ferreira-Marques ${ }^{20}$, W Furman ${ }^{25}$, I Goncalves ${ }^{20}$, \\ E Gonzalez-Romero ${ }^{10}$, F Gramegna ${ }^{17}$, C Guerrero ${ }^{10}$, F Gunsing ${ }^{6}$, \\ B Haas ${ }^{26}$, R Haight ${ }^{27}$, A Herrera-Martinez ${ }^{4}$, M Igashira ${ }^{28}$, E Jericha ${ }^{13}$, \\ Y Kadi ${ }^{4}$, D Karadimos ${ }^{12}$, D Karamanis ${ }^{12}$, M Kerveno ${ }^{14}$, P Koehler ${ }^{29}$, \\ E Kossionides ${ }^{30}$, M Krtička ${ }^{15}$, C Lamboudis ${ }^{23}$, H Leeb ${ }^{13}$, A Lindote Le, $^{20}$ \\ I Lopes $^{20}$, M Lozano ${ }^{18}$, S Lukic ${ }^{14}$, J Marganiec ${ }^{11}$, S Marrone ${ }^{7}$,

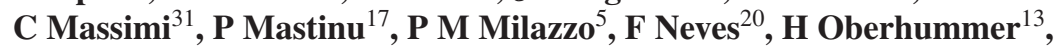 \\ S O'Brien ${ }^{22}$, J Pancin', C Papachristodoulou ${ }^{12}$, C Papadopoulos ${ }^{32}$, \\ C Paradela ${ }^{9}$, N Patronis ${ }^{12}$, A Pavlik ${ }^{14}$, P Pavlopoulos ${ }^{33}$, L Perrot ${ }^{6}$,

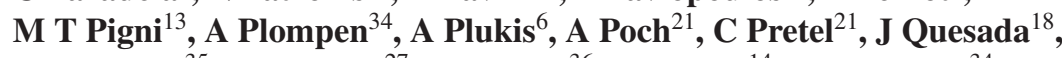 \\ T Rauscher $^{35}$, R Reifarth ${ }^{27}$, C Rubbia ${ }^{36}$, G Rudolf ${ }^{14}$, P Rullhusen ${ }^{34}$, \\ J Salgado $^{19}$, C Santos ${ }^{19}$, L Sarchiapone ${ }^{4}$, I Savvidis ${ }^{23}$, C Stephan ${ }^{24}$, \\ G Tagliente ${ }^{7}$, J L Tain ${ }^{37}$, L Tassan-Got ${ }^{24}$, L Tavora ${ }^{19}$, G Vannini ${ }^{31}$,

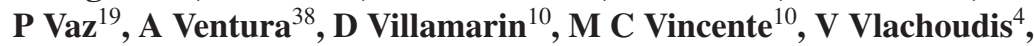

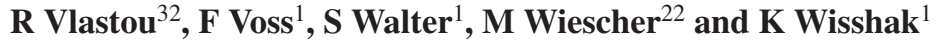 \\ (The n_TOF Collaboration)
}

\footnotetext{
${ }^{1}$ Forschungszentrum Karlsruhe GmbH (FZK), Institut für Kernphysik, Germany

${ }^{2}$ Gesellschaft für Schwerionenforschung mbH, Darmstadt, Germany

${ }^{3}$ International Atomic Energy Agency (IAEA), NAPC/Nuclear Data Section, Vienna, Austria

${ }^{4}$ CERN, Geneva, Switzerland

5 Istituto Nazionale di Fisica Nucleare (INFN), Trieste, Italy

${ }^{6}$ CEA/Saclay-DSM/DAPNIA, Gif-sur-Yvette, France

${ }^{7}$ Istituto Nazionale di Fisica Nucleare (INFN), Bari, Italy

${ }^{8}$ Università degli studi di Torino, Torino, Italy

${ }^{9}$ Universidade de Santiago de Compostela, Spain

${ }^{10}$ Centro de Investigaciones Energeticas Medioambientales y Tecnologicas, Madrid, Spain

${ }^{11}$ University of Lodz, Lodz, Poland

12 University of Ioannina, Greece

13 Atominstitut der Österreichischen Universitäten, Technische Universität Wien, Austria

${ }^{14}$ Centre National de la Recherche Scientifique/IN2P3-IReS, Strasbourg, France

${ }^{15}$ Charles University, Prague, Czech Republic

${ }^{16}$ Universitat Politecnica de Catalunya, Barcelona, Spain

${ }^{17}$ Istituto Nazionale di Fisica Nucleare (INFN), Laboratori Nazionali di Legnaro, Italy

${ }^{18}$ Universidad de Sevilla, Spain

${ }^{19}$ Instituto Tecnológico e Nuclear (ITN), Lisbon, Portugal

${ }^{20}$ LIP - Coimbra and Departamento de Fisica da Universidade de Coimbra, Portugal

${ }^{21}$ Universitat Politecnica de Catalunya, Barcelona, Spain
} 
${ }^{22}$ University of Notre Dame, Notre Dame, IN 46556, USA

${ }^{23}$ Aristotle University of Thessaloniki, Greece

${ }^{24}$ Centre National de la Recherche Scientifique/IN2P3-IPN, Orsay, France

${ }^{25}$ Joint Institute for Nuclear Research, Frank Laboratory of Neutron Physics, Dubna, Russia

${ }^{26}$ Centre National de la Recherche Scientifique/IN2P3-CENBG, Bordeaux, France

${ }^{27}$ Los Alamos National Laboratory, NM 87545, USA

${ }^{28}$ Tokyo Institute of Technology, Tokyo, Japan

${ }^{29}$ Oak Ridge National Laboratory, Physics Division, Oak Ridge, TN 37831, USA

${ }^{30}$ NCSR, Athens, Greece

${ }^{31}$ Dipartimento di Fisica, Università di Bologna, and Sezione INFN di Bologna, Italy

${ }^{32}$ National Technical University of Athens, Greece

${ }^{33}$ Pôle Universitaire Léonard de Vinci, Paris La Défense, France

${ }^{34}$ CEC-JRC-IRMM, Geel, Belgium

${ }^{35}$ Department of Physics and Astronomy, University of Basel, Basel, Switzerland

${ }^{36}$ Università degli Studi di Pavia, Pavia, Italy

${ }^{37}$ Instituto de Física Corpuscular, CSIC-Universidad de Valencia, Spain

${ }^{38}$ ENEA, Bologna, Italy

E-mail: marita.mosconi@ik.fzk.de

Received 29 June 2007

Published 13 December 2007

Online at stacks.iop.org/JPhysG/35/014015

\section{Abstract}

Dating the age of the universe by the $\beta$-decay of ${ }^{187} \operatorname{Re}\left(t_{1 / 2}=41.2 \mathrm{Gyr}\right)$ requires the accurate assessment of the $s$-process reaction flow. The daughter nucleus ${ }^{187}$ Os as well as its immediate $s$-only neighbor ${ }^{186}$ Os are shielded from the $r$-process. Therefore, the radiogenic contribution to ${ }^{187} \mathrm{Os}$ can be derived via the local approximation of the $s$-process, which is well justified in this mass region: $\left(\langle\sigma\rangle \mathrm{N}_{s}\right)_{187}=\left(\langle\sigma\rangle \mathrm{N}_{s}\right)_{186}$. Accordingly, accurate Maxwellianaveraged $(n, \gamma)$ cross sections have to be known for ${ }^{186}$ Os and ${ }^{187}$ Os over the full range of $s$-process temperatures. Because ${ }^{187}$ Os has low-lying excited states, which are significantly populated during the $s$-process, neutron captures on these excited states have to be properly taken into account. The input for the corresponding Hauser-Feshbach calculations can be constrained by deriving the strength functions from a detailed resonance analysis of the measured $(n, \gamma)$ cross sections as well as by an additional measurement of the inelastic scattering cross section for the first exited level, which dominates the competition between the capture and scattering channels. This contribution reports on measurements of the neutron capture cross sections of ${ }^{186} \mathrm{Os},{ }^{187} \mathrm{Os}$ and ${ }^{188} \mathrm{Os}$ from the $\mathrm{eV}$ region to $1 \mathrm{MeV}$ at the CERN/n_TOF facility and of the inelastic scattering cross section of ${ }^{187} \mathrm{Os}$ around $30 \mathrm{keV}$ at the Karlsruhe 3.7 MV Van de Graaff accelerator. Based on these results and on a detailed resonance analysis, improved Maxwellian-averaged cross sections were obtained for thermal energies between $5 \mathrm{keV}$ and $100 \mathrm{keV}$. The implications of these results for the Re/Os clock are discussed.

(Some figures in this article are in colour only in the electronic version) 


\section{Introduction}

The decay of ${ }^{187} \operatorname{Re}\left(t_{\frac{1}{2}}=41.2\right.$ Gyr [1]) can be considered as a chronometer for the $r$-process. The particularity of this clock is that the daughter ${ }^{187} \mathrm{Os}$ is synthesized otherwise only by the $s$-process, and that its radiogenic component can be separated by means of $s$-process systematics. Consequently, the history of the ${ }^{187} \mathrm{Re}$ enrichment in the galaxy is important. This makes this cosmochronometer complementary to the ${ }^{238} \mathrm{U} /{ }^{232} \mathrm{Th}$ clock that requires the primordial $r$-process yields, but does not depend on a galactic chemical evolution model [2]. Calculations of $s$-process abundances depend on accurate $(n, \gamma)$ cross sections averaged over the stellar neutron energy distribution and over the thermally populated nuclear levels. Therefore, the measurement of these cross sections must be complemented by calculations with the Hauser-Feshbach statistical model (HFSM) to take the effect of the excited states into account. In this context, the first excited state in ${ }^{187} \mathrm{Os}$ at $9.75 \mathrm{keV}$ is important because it is strongly populated at thermal energies typical of the $s$-process in asymptotic giant branch (AGB) stars [3]. Theoretical calculations of neutron capture cross section for excited states can be considerably improved if the relevant parameters are determined experimentally. This holds for the level densities and strength functions, which were deduced in the present analysis of the resolved resonances, and for the neutron transmission functions, which can be constrained via the inelastic cross section. The second aspect applies also to the treatment of the so-called super-(in)elastic scattering channels, where neutrons scatter on excited states and gain in energy by de-excitation of the scattering state. Previous evaluations of the Re/Os chronometer suffered from an uncertainty of about $15 \%$ in the derived age due to discrepancies in the stellar $(n, \gamma)$ rates for ${ }^{186}$ Os and ${ }^{187}$ Os from experiments [4-8] and calculations [9, 10]. Therefore, improved measurements of the $(n, \gamma)$ cross sections of ${ }^{186}$ Os and ${ }^{187}$ Os as well as of the $\left(n, n^{\prime}\right)$ cross section of ${ }^{187}$ Os have been performed at the $n_{-}$TOF spallation facility at CERN and at the Karlsruhe 3.7 MV Van de Graaff accelerator.

\section{Radiative neutron capture cross sections}

Previous $(n, \gamma)$ cross sections for ${ }^{186,187}$ Os were reported in limited energy ranges only, from $2.75 \mathrm{keV}$ to $2.65 \mathrm{MeV}$ [4] and from $1 \mathrm{keV}$ to $135 \mathrm{keV}$ [6]. These data were in good agreement for ${ }^{187}$ Os but showed discrepancies for ${ }^{186}$ Os. A theoretical calculation based on the available total cross sections [11], level densities and strength functions [12] yields a different behavior compared to both measurements, as shown in the inset of figure 1 . Moreover, experimental information below $1 \mathrm{keV}$ is rather incomplete [13], leading to large uncertainties in the calculation of Maxwellian-averaged cross sections (MACS) at the low thermal energies. Experimental setup and techniques used for the $(n, \gamma)$ cross section measurements on ${ }^{186,187,188}$ Os at the n_TOF spallation facility [14] are described in [15-17]. In the following, we will focus on the relevant aspects of the final analysis. The raw data were treated by the pulse height weighting technique (PHWT) $[18,19]$, and the analysis of the resulting reaction yield was performed separately in the resolved and unresolved resonance region. The cross section in the continuum was extracted after background subtraction and flux normalization, with additional corrections for multiple scattering (obtained by the SESH code [23]) and for isotopic impurities [15]. The flux was normalized in two ways, via the experimental MACS of gold at $k T=25 \mathrm{keV}$ [22] and independently via the saturated $4.9 \mathrm{eV}$ resonance of the gold sample [13]. The two methods were in agreement and a systematic uncertainty of $2 \%$ has been evaluated for the flux shape by comparing the results from different neutron detectors. Below $2-3 \mathrm{keV}$ the individual resonance parameters have been extracted [20] by means of the $R$-matrix code SAMMY [24]. The final cross 

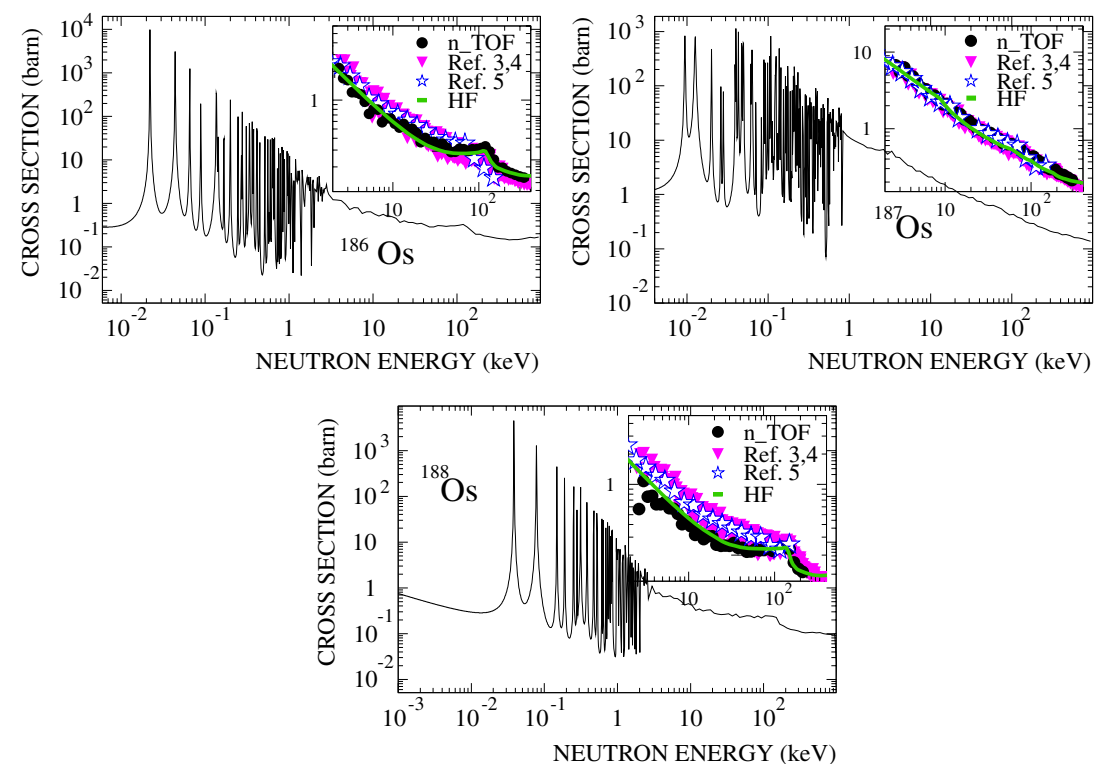

Figure 1. The measured Os cross sections between eV region and $1 \mathrm{MeV}$. The energy averaged cross section in the insets is partly obtained from the resonance parameters (below $3 \mathrm{keV}$ in case of ${ }^{186,188} \mathrm{Os}$ and below $2 \mathrm{keV}$ for ${ }^{187} \mathrm{Os}$ ). Comparison with previous data in the insets shows significant discrepancies for the even isotopes (see the text).

Table 1. The MACS (in mb) based on the experimental results at $\mathrm{n}_{-}$TOF compared with previous data at the reference energy of $k T=30 \mathrm{keV}$.

\begin{tabular}{lcll}
\hline & ${ }^{186}$ Os & ${ }^{187}$ Os & ${ }^{188}$ Os \\
\hline This work & $410 \pm 17$ & $966 \pm 31$ & $293 \pm 14$ \\
{$[25]$} & & $922 \pm 46$ & \\
{$[6]$} & $438 \pm 30$ & $919 \pm 43$ & $395 \pm 24$ \\
{$[4,5]$} & $418 \pm 16$ & $874 \pm 28$ & $401 \pm 15$ \\
{$[26]$} & $422 \pm 16$ & $896 \pm 30$ & $399 \pm 15$ \\
\hline
\end{tabular}

section was then determined by combining the analyses for the resonance region and the continuum. The results show good agreement with the theoretical prediction based on available experimental data, but deviate from previous measurements in case of the even isotopes ${ }^{186} \mathrm{Os}$ and ${ }^{188}$ Os (figure 1). The present analysis was repeatedly performed by independent methods to ensure that systematic uncertainties were well under control. For example, the consistent data obtained in the overlap region of the resonance analysis, where the background was evaluated by the SAMMY fits, and of the results at the border of the unresolved region confirmed the background determination around a few $\mathrm{keV}$. At higher energies, the background has been further verified by measurements with the black neutron filters [15] and by comparison with detailed GEANT simulations [21]. The respective MACS at $k T=30 \mathrm{keV}$ were found to agree within 3\% for all three isotopes. The effect of the weighting functions was studied by a set of GEANT simulations of the experimental setup with plausible variations of the corresponding parameters. The so-obtained weighting functions were almost identical, leading to cross section differences of $\leqslant 2 \%$. This value was, therefore, adopted as the intrinsic uncertainty of the PHWT [19]. The MACS for the measured ground state cross sections are summarized in table 1 . 


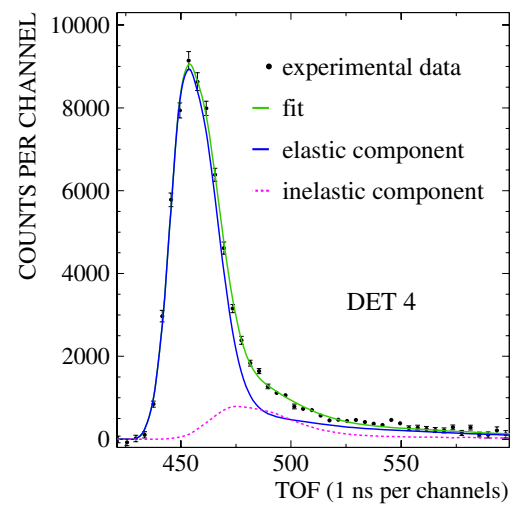

Figure 2. Fit to the TOF spectrum of scattered neutrons, illustrating the separation of the elastic and inelastic components. The only free parameters are the amplitudes of the two components, since their relative position is defined by the excitation energy of the scattering state at $9.75 \mathrm{keV}$. The error bars on the data points correspond to the systematic uncertainties due to background subtraction (statistical uncertainties are smaller than $1 \%$.

\section{Inelastic scattering cross section}

The inelastic scattering cross section to the first excited level of ${ }^{187}$ Os was previously measured with uncertainties between $13 \%$ and $30 \%$, using filtered neutron beams $[7,8,27]$ and - in a single case-a quasi-monoenergetic beam of $60 \mathrm{keV}$ produced by the ${ }^{7} \mathrm{Li}(p, n)^{7} \mathrm{Be}$ reaction [28]. The latter technique has also been chosen for an additional measurement at Karlsruhe, aiming at improved accuracy and at producing a distribution with $E_{n}=30 \mathrm{keV}$, closer to the relevant astrophysical energy. In different runs, Gaussian-like neutron spectra could be produced at the threshold of the ${ }^{7} \mathrm{Li}(p, n){ }^{7} \mathrm{Be}$ reaction with an FWHM from 7.6 to $9.5 \mathrm{keV}$, sufficient for separating the elastic and inelastic components in the spectra of scattered neutrons. The proton beam was pulsed with a repetition rate of $1 \mathrm{MHz}$ and a pulse width of $10 \mathrm{~ns}$. The scattering sample $(1.5 \mathrm{~cm}$ in diameter, $2 \mathrm{~g}$ in mass) was placed $4 \mathrm{~cm}$ from the lithium target at an angle of $45^{\circ}$ with respect to the proton beam. The neutron energy was measured via the time-of-flight (TOF) technique, using a flight path of $26.1 \mathrm{~cm}$ between the sample and three neutron detectors placed at $90^{\circ}, 120^{\circ}$ and $270^{\circ}$. The shape of the elastic TOF distribution has been obtained by a detailed GEANT simulation and was validated by the pure elastic yield from an ${ }^{188} \mathrm{Os}$ sample, while that of the inelastic component was obtained relative to the elastic part by the energy shift corresponding to the excitation energy of the scattering state at $9.75 \mathrm{keV}$. This analysis replaces a preliminary fit [17] and confirms the alternative approach reported in $[16,29]$. The separation of the two components in the TOF spectrum of ${ }^{187}$ Os is shown in figure 2 for one of the detectors. The GEANT simulation of the experiment resulted in a robust and stable analysis of the various runs, which allowed us to determine the elastic/inelastic ratio with an accuracy of $6 \%$. If the inelastic cross section derived from this result is used to normalize a previous HFSM calculation, one finds fair agreement with existing data at higher energies [7, 27, 28] as shown in figure 3. Additional information on the measurement and data analysis has been reported in [30].

\section{Stellar neutron capture rates and astrophysical implications}

With the information from the $(n, \gamma)$ and $\left(n, n^{\prime}\right)$ measurements, a complete and consistent parameter set for the HFSM calculations of the MACS could be established for the 


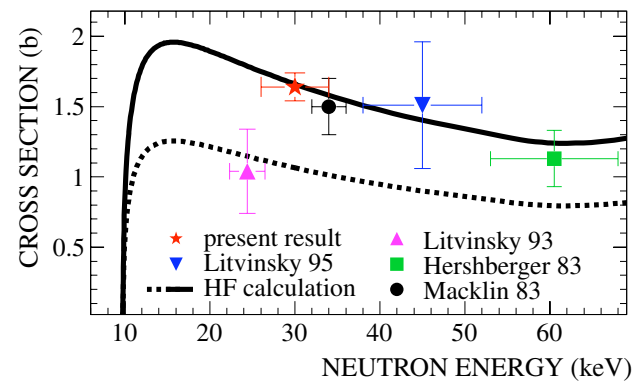

Figure 3. Inelastic scattering cross section to the first excited state in ${ }^{187}$ Os. Normalization of the theoretical prediction (dotted line) to the experimental results is in fair agreement with most experimental data.

first time. In particular, average level spacings and gamma-ray decay widths of s-wave neutron resonances, neutron strength functions, capture cross sections for the target ground state, and $\left(n, n^{\prime}\right)$ cross section data have been used to constrain the HFSM calculations, assuming a spherical shape for the optical model interaction, as well as assuming deformed target states (coupled-channel calculations). A detailed account of this analysis will be reported elsewhere [31]. Here, we summarize the conclusion of this study which, by the improvement of the $(n, \gamma)$ and of the inelastic cross section, allowed to reduce the contribution of the nuclear data input (including the effect of excited states) to the uncertainty of the Re/Os clock to a level of about 7\% (1 Gyr for an age of $15 \mathrm{Gyr}$ ), where it no longer impedes further analysis of the astrophysical aspects. The largest remaining uncertainties of the Re/Os clock should be related to Galactic chemical evolution modeling and to astration effects [32].

\section{References}

[1] Galeazzi M, Fontanelli F, Gatti F and Vitale S 2000 Phys. Rev. C 63014302

[2] Cowan J J, Pfeiffer B, Kratz K-L, Thielemann F-K, Sneden C, Burles S, Tytler D and Beers T C 1999 Astrophys. J. 521194

[3] Busso M, Gallino R and Wasserburg G J 1999 Ann. Rev. Astron. Astrophys. 37239

[4] Winters R R, Macklin R L and Halperin J 1980 Phys. Rev. C 21563

[5] Winters R R and Macklin R L 1982 Phys. Rev. C 25208

[6] Browne J C and Berman B L 1981 Phys. Rev. C 231434

[7] Macklin R L, Winters R R, Hill N W and Harvey J A 1983 Astrophys. J. 274408

[8] Litvinsky L L 1993 Phys. At. Nuclei 5617

[9] Goriely S 1997 Nuclear Data for Science and Technology ed G Reffo, A Ventura and C Grandi (Trieste: Italian Physical Society) p 811

[10] Rauscher T and Thielemann F-K 2000 At. Data Nucl. Data Tables 751

[11] ENDF Library 2002 Brookhaven National Nuclear Data Center (http://www.nndc.bnl.gov/)

[12] Mughabghab S F 1984 Neutron Cross Sections (New York: Academic)

[13] Mughabghab S F 2006 Atlas of Neutron Resonances (New York: Elsevier)

[14] Abbondanno U et al 2002 Technical Report CERN/INTC 2002-037 (CERN, Geneva)

[15] Mosconi M et al 2005 Nuclear Data for Science and Technology ed R C Haight, M B Chadwick, T Kawano and P Talou (New York: AIP) p 1335

[16] Mosconi M et al 2006 Nuclei in the Cosmos-IX ed A Mengoni (Trieste: Proceedings of Science) p 055 http://pos.sissa.it

[17] Mosconi M et al 2007 Prog. Part. Nucl. Phys. 55165

[18] Aerts G Berthoumiex E Gunsing F and Perrot L 2004 Technical Report DAPNIA-04-106 (CEA Saclay)

[19] Abbondanno U et al 2004 Nucl. Instrum. Methods A $\mathbf{5 2 1} 454$ 
[20] Fujii K et al 2007 Proc. Int. Conf. on Nuclear Data for Science and Technology (CEA, Cadarache, April 22-27)

[21] Apostolakis J 1993 CERN Program Library (CERN, Geneva), http://wwwinfo.cern.ch/asd/geant/

[22] Ratynski W and Käppeler F 1988 Phys. Rev. C 37595

[23] Fröhner F H 1968 Technical report GA-8380 (Gulf General Atomic, San Diego)

[24] Larsen N 2003 Updated Users' Guide for SAMMY: ORNL/TM-9179/R6 (Oak Ridge: Oak Ridge National Laboratory)

[25] Bokhovko M V, Kononov V N, Poletaev E D, Rabotnov N S and Timokhov V M 1992 Nuclear Datafor Science and Technology ed S M Qaim (Berlin: Springer) p 62

[26] Bao Z Y, Beer H, Käppeler F, Voss F, Wisshak K and Rauscher T 2000 At. Data Nucl. Data Tables 7670

[27] Litvinsky L L, Zhigalov Ya A, Libman V A, Murzin A V and Shkarupa A M 1995 Phys. At. Nuclei 58164

[28] Hershberger R L, Macklin R L, Balakrishnan M, Hill N W and McEllistrem M T 1983 Phys. Rev. C 282249

[29] Mosconi M, Plag R, Heil M, Käppeler F and Mengoni 2007 Proc. 8th Int. Topical Meeting on Nuclear Applications and Utilization of Accelerators (ANS La Grange Park, IL, USA, July 29-Aug. 2)

[30] Mosconi M, Heil M, Käppeler F, Mengoni A and Plag R 2007 Proc. Int. Conf. on Nuclear Data for Science and Technology (CEA Cadarache, April 22-27)

[31] Abbondanno U et al (The n_TOF Collaboration) in preparation

[32] Takahashi K 2003 Nucl. Phys. A 718325 